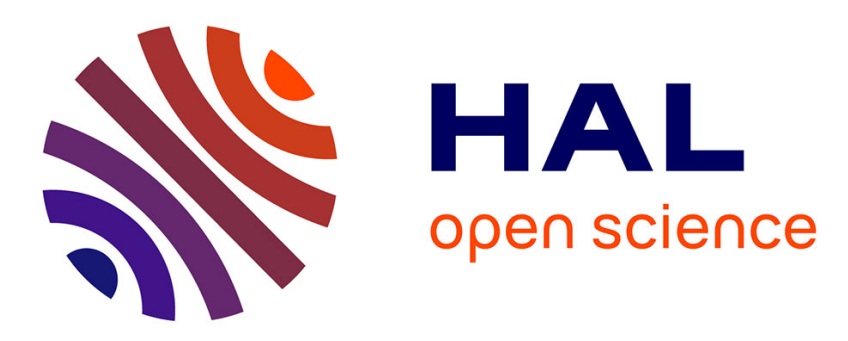

\title{
Stimulated Raman scattering: Action evolution and particle trapping via Euler-Vlasov fluid simulation
}

T. W. Johnston, Pierre Bertrand, A. Ghizzo, M. Shoucri, E. Fijalkow, M. R.

Feix

\section{- To cite this version:}

T. W. Johnston, Pierre Bertrand, A. Ghizzo, M. Shoucri, E. Fijalkow, et al.. Stimulated Raman scattering: Action evolution and particle trapping via Euler-Vlasov fluid simulation. Physics of Fluids B: Plasma Physics (1989-1993) , 1992, 4 (8), pp.2523-2537. 10.1063/1.860168 . hal-01791842

\section{HAL Id: hal-01791842 \\ https://hal.univ-lorraine.fr/hal-01791842}

Submitted on 14 May 2018

HAL is a multi-disciplinary open access archive for the deposit and dissemination of scientific research documents, whether they are published or not. The documents may come from teaching and research institutions in France or abroad, or from public or private research centers.
L'archive ouverte pluridisciplinaire HAL, est destinée au dépôt et à la diffusion de documents scientifiques de niveau recherche, publiés ou non, émanant des établissements d'enseignement et de recherche français ou étrangers, des laboratoires publics ou privés. 


\section{Stimulated Raman scattering: Action evolution and particle trapping via Euler- Vlasov fluid simulation}

T. W. Johnston, P. Bertrand, A. Ghizzo, M. Shoucri, E. Fijalkow, and M. R. Feix

Citation: Physics of Fluids B: Plasma Physics 4, 2523 (1992); doi: 10.1063/1.860168

View online: https://doi.org/10.1063/1.860168

View Table of Contents: http://aip.scitation.org/toc/pfb/4/8

Published by the American Institute of Physics

\section{Articles you may be interested in}

Theory of stimulated scattering processes in laser-irradiated plasmas

The Physics of Fluids 18, 1002 (1975); 10.1063/1.861248

Electron holes in phase space: What they are and why they matter

Physics of Plasmas 24, 055601 (2017); 10.1063/1.4976854

Structure and structure-preserving algorithms for plasma physics

Physics of Plasmas 24, 055502 (2017); 10.1063/1.4982054

A hybrid semi-Lagrangian Vlasov code. II. Numerical simulation of chirped beatwave experiment

Physics of Plasmas 5, 4041 (1998); 10.1063/1.873126

A time-implicit numerical method and benchmarks for the relativistic Vlasov-Ampere equations

Physics of Plasmas 23, 012102 (2016); 10.1063/1.4938035

Resonance between heat-carrying electrons and Langmuir waves in inertial confinement fusion plasmas Physics of Plasmas 23, 012707 (2016); 10.1063/1.4939603 


\title{
Stimulated Raman scattering: Action evolution and particle trapping via Euler-Vlasov fluid simulation
}

\author{
T. W. Johnston \\ INRS-Energie, C.P. 1020, Varennes, Québec J3X 1S2, Canada \\ P. Bertrand and A. Ghizzo \\ Physique Théorique, Université de Nancy I, Nancy, France \\ M. Shoucri \\ Hydro-Québec, Groupe TAI Vice-Présidence Recherche IREQ C.P. 1020, Varennes, \\ Québec J3X ISI, Canada \\ E. Fijalkow and M. R. Feix \\ PMM-CNRS, Université d'Orléans, Orléans, France
}

(Received 23 March 1992; accepted 4 May 1992)

\begin{abstract}
The Manley-Rowe action relations and trapped-electron orbit theory have been applied to the problem of stimulated Raman scattering (SRS) in the forward direction, as simulated by an Euler-Vlasov fluid (EVF) code in periodic geometry. After a slight initial delay, the Vlasov results agree well with three-oscillator theory (but with damping larger than the Landau linear value). The action sum of the pump and the scattered SRS wave was well conserved, while the action loss between the pump and plasma wave was shown to be well accounted for in detail by examining the electron energy for electrons above the lower trapping boundary (separatrix) in $x$ - $p_{x}$ phase space. However, unlike the three-oscillator model, the action sum of the pump and plasma wave was observed to actually increase for a short time because of retrieval of apparently lost action from the trapped-electron effect in regenerating the plasma wave. Thus the utility was clearly demonstrated of using action conservation to separate the effects of three-wave interaction from those due to nonlinear particle-wave interaction. The EVF code also shows clearly the fine details of layering and folding seen in $x-p_{x}$ phase space due to electron trapping, detrapping, and retrapping. [These details would have been invisible in the usual particle-in-cell (PIC) code because of graininess at low phase space densities.]
\end{abstract}

\section{INTRODUCTION}

The acceleration of charged particles by fast $(\omega /$ $k c \sim 1)$ large-amplitude longitudinal electron plasma wave trains has received considerable attention in recent years. One of the potentially most effective ways to produce such a wave uses the forward stimulated Raman scattering ${ }^{1}$ (SRS) process (here denoted by FRS, for forward Raman scattering), long of interest for laser fusion. As is well known, this is a stimulated decay of incident light into a forward-scattered light wave and a forward-going plasma wave (necessarily at plasma densities below the pump frequency quarter-critical value). In the driven version of this process, the beat wave acceleration scheme ${ }^{2}$ relies upon the nonlinear resonant interaction of two parallel intense laser beams [pump $\left(\omega_{0}, k_{0}\right)$, and idler $\left(\omega_{s}, k_{s}\right)$ ] at frequencies $\omega_{0}$ and $\omega_{s}$, with frequency difference close to $\omega_{p}$, the plasma frequency, and chosen more exactly to be equal to the Bohm-Gross electron plasma wave frequency $\omega_{e}$ for wave number $k_{e}=k_{0}-k_{s}$

Several numerical simulations $s^{3-5}$ have been performed to investigate the details of the plasma wave trapping dynamics responsible for the acceleration of electrons. However, these simulations, employing as they do the wellknown (macro-) particle-in-cell (PIC) technique, have difficulty in supplying a usefully precise description of the electron acceleration process. This is because the PIC codes lack enough simulation particles to display the detailed phase-space structure of the distribution function which is often obtained in those regions of phase space where particle and phase velocities are comparable and where trapping occurs. If more than one space dimension is involved, only the PIC code is practicable so far. However, when one space dimension will do, the Euler-Vlasov fluid codes (where one calculates the Vlasov fluid density in phase space without the use of simulation macroparticles) have been found to be a powerful tool for studying in detail the particle acceleration dynamics (relativistic in this case) both in a conveniently short but idealized periodic ${ }^{6,7}$ plasma and in the more realistic long, open system $^{8}$ with external sources, which we characterize as "causal."

The two methods of simulation are complementary. Roughly speaking, PIC codes are relatively efficient and readily scalable to two and three dimensions, but these codes are noisy and lack detail in low-density regions of phase space because of the "graininess" of the simulation macroparticles. In one degree of freedom, the EulerVlasov fluid (EVF) method is usually comparable in computer loading, is noiseless, and is probably much easier to realize in massively parallel computers (because one does not have to cope with the allocation of the particles between processors, since the Euler elements stay in place). 
Unfortunately the EVF computer loading rises dramatically if extended to more dimensions. (One calculates for large phase space regions with negligible density, often because one must ensure that all of the phase space is covered, since unforeseen acceleration beyond prescribed momentum limits means a loss of plasma from the computation phase space.) It is for this reason that, in our one-and-one-half dimensions ( $\left.1 \frac{1}{2}-\mathrm{D}\right)$ work, the transverse velocity effects are approximated by momentum conservation of a transversely cold fluid. (We have verified that the full $1 \frac{1}{2}-D$ EVF, which runs much more slowly, gives results $^{6}$ that are only very slightly different for our problem.) Nonetheless, if fine details and low noise are required, the Euler-Vlasov method has no rival.

In this paper, we use this EVF phase space resolution to advantage in order to account in detail for the action loss in a periodic-plasma simulation of an initial-value forward SRS instability, allowing close comparison with the canonical three-oscillator analysis. The presentation of this work is as follows. In Sec. II, the code is discussed, as well as some comparisons between periodic boundary conditions and nonperiodic boundary conditions, together with three-wave resonance considerations that determine some of the modeling and simulation parameters. Next, in Sec. III, we discuss the relevant parametric coupling theory, first for the nonlinear three-oscillator model (which includes linear loss, linear mismatch, and first-order relativistic detuning) and then for the initial linear development. (Some of the details are relegated to Appendices A and B.) After that, in Sec. IV, the preceding analysis is applied to choose the conditions for the EVF simulation, followed by the simulation results themselves. The EVF simulation results on action evolution are compared, in Sec. V, with the results of Sec. IV. Features of trapping dynamics are considered in detail in Sec. VI, including phase space density structures and separatrices. Section VII contains some discussion and the conclusions. The two specialized topics left for Appendices A and B are, respectively, the detailed derivation of the action form of the coupled-wave model for SRS and the initial-value coupled-mode solution pairs for the linear instability problem.

\section{THE RELATIVISTIC EULER-VLASOV FLUID CODE AND SRS}

In order to handle the plasma wave velocities relevant to future particle accelerators, the model equation must at least be relativistic in the acceleration direction $(x)$. For the linear ( $y$-direction) laser polarization intensities we consider here, the transverse dynamics can be economically included in the nonrelativistic transverse cold-fluid approximation. The Vlasov equation in the acceleration $(x)$ direction is thus

$$
\frac{\partial f}{\partial t}+\frac{p_{x}}{m \gamma} \frac{\partial f}{\partial x}-e\left(E_{x}+v_{y} B_{z}\right) \frac{\partial f}{\partial p_{x}}=0
$$

where $\gamma=\left(1+p_{x}^{2} / m^{2} c^{2}\right)$. In the $y$ direction, for reasons of computer economy, the cold nonrelativistic $\left(p_{y} \approx m v_{y}\right)$ approximation is used:

$$
\frac{\partial v_{y}}{\partial t}=-\frac{e}{m} E_{y}
$$

(As mentioned above, we did verify ${ }^{6}$ that this approximation is indeed valid.)

These two equations, together with the usual onedimensional $(x)$ Maxwell equations, make up the system of equations used here. The details of the way in which the equations are advanced and the boundary conditions are imposed have been extensively described elsewhere, ${ }^{6-8}$ but nonetheless we find it useful here to recapitulate some of the discussion of boundary conditions for the initial-value space periodic case.

While the demanding causa ${ }^{8}$ simulations are necessary to understand realistic cases, these simulations are also difficult to analyze in detail sufficient to gain even qualitative physical understanding. One of the difficulties is that even the simplest coupled wave models (say with nonlinear plasma particle effects absorbed into an effective linear damping or ignored completely) involve coupled nonlinear partial differential equations in both time and space, involving different group velocities. For these cases, even a concept so basic as action sum conservation is very awkward to use. To assist in the development and testing of such basic concepts, the much more limited and less demanding spatially periodic simulations of evolution from an initial state can be very useful, since now one needs to consider the behavior in time only of the discrete spatial Fourier modes. In the coupled wave model for the SRS parametric instability, this allows one to, in effect, drop the group-velocity-times-spatial-gradient terms, convert to ordinary coupled differential equations, and analyze action density conservation. Looking at the action change in lossless channels (such as the electromagnetic ones) allows one to separate the effects of nonlinear damping (particle trapping, etc.) from nonlinear wave generation. In this paper, we will discuss a periodic EVF simulation of a particular FRS case and its three-oscillator model.

In the one-dimensional spatially periodic simulation, particles and waves exiting either spatial boundary enter the other. Clearly, a periodic simulation will be significantly less demanding than a fully causal simulation only if the spatial period (the largest spatial wavelength) is much less than the size of the calculation length in a fully spatiotemporally causal calculation of useful size. This smaller spatial period necessarily means a coarsening of the allowed wave-vector spectrum. In order to see waves most clearly, the lower excited mode number should be 2 (i.e., two wavelengths in the simulation box), while the others should be in the ratio of reasonably small whole-number ratios, to keep the computational load to a minimum.

Naturally, the investigation of any problem in a small box must be carefully tailored to avoid artificially suppressing some particular physical effect because the appropriate wave number is not sufficiently close to one permitted by the periodic box condition. For three-wave instabilities at least (and almost certainly also for the more complicated cases such as, say, the anti-Stokes wave), the linear dispersion analysis and tests with the three-oscillator model show 
that any $\omega$ mismatch imposed by the finite spatial grid results in some growth reduction [see Eq. (11) and the related discussion] as the system adjusts itself to achieve exact frequency matching and phase locking. If, however, one makes a careful choice of the free parameters (in this case the plasma parameters) so as to keep the square of the frequency mismatch small compared with the square of the growth rate term, the results are only slightly changed from the near-perfect match attainable in a very large box. To do this, one must use quite accurate dispersion relations for the waves concerned.

For our forward scattering geometry the wave-number choices were $k$ (pump) $\sim$ (mode 4$)$ to he equal to $k$ (scattered) $\sim$ (mode 2$)+k$ (plasma wave) $\sim$ (mode 2$)$. The initial conditions and plasma parameters were then chosen (as discussed in Sec. IV) with reference to perturbation theory so as to satisfy frequency resonance as closely as feasible at a useful plasma temperature.

We now turn to the relevant theory, i.e., to small signal growth, resonance, and damping and also to action sum conservation and evolution.

\section{SRS AND THE MANLEY-ROWE RELATIONS}

\section{A. Manley-Rowe relations and linear behavior}

In the three-wave parametric decay from the pump wave " 0 " to the scattered wave " $s$ " and the lossy (electron plasma) wave "e," we have, as usual,

$$
\omega_{0}\left(k_{0}\right)+\Delta \omega=\omega_{s}\left(k_{s}\right)+\omega_{e}\left(k_{e}\right), \quad k_{0}=k_{s}+k_{e},
$$

we assume perfect $k$ matching (since we have a periodic simulation and match mode numbers exactly), while $\Delta \omega$ contains the mismatch (if any).

Because we eventually wish to discuss action conservation, we found it convenient to develop the fluid model results at first in terms of field variables (for which the energy density is easy to write), namely, the vector potential $A_{y}$ for the transverse waves and the electric field $E_{x}$ (rather than the more usual density perturbation ${ }^{9,10}$ ) for the electrostatic waves. The derivation is given in Appendix $\mathrm{A}$ and the unnormalized results are as follows:

$$
\begin{aligned}
& \left(\frac{\partial}{\partial t}+v_{g 0} \frac{\partial}{\partial x}\right) A_{0}=-\frac{e}{4 m} \cdot \frac{k_{e}}{\omega_{0}} A_{s} E_{e} e^{+i \Delta \omega t}, \\
& \left(\frac{\partial}{\partial t}+v_{g s} \frac{\partial}{\partial x}\right) A_{s}=\frac{e}{4 m} \cdot \frac{k_{e}}{\omega_{0}} A_{0} E_{e}^{*} e^{-i \Delta \omega t}, \\
& {\left[\frac{\partial}{\partial t}+\gamma+v_{g s} \frac{\partial}{\partial x}+i \frac{3}{8}\left(\frac{e}{m c}\right)^{2} \frac{\omega_{e}^{3}}{\omega_{p}^{4}}\left|E_{e}\right|^{2}\right] E_{e}} \\
& \quad=\frac{e}{4 m} \frac{k_{e}}{\omega_{e}} \omega_{p}^{2} A_{0} A_{s}^{*} e^{-i \Delta \omega t} .
\end{aligned}
$$

Here, as usual,

$$
\begin{aligned}
& A_{y 0,5}(x, t)=\operatorname{Re}\left(A_{0,5} e^{-i\left(\omega_{0,5} t-k_{0,5} x\right)}\right), \\
& E_{x e}(x, t)=\operatorname{Re}\left(E_{e} e^{-i\left(\omega_{e} t-k_{e} x\right)}\right),
\end{aligned}
$$

and $A_{0}, A_{s}$, and $E_{e}$ are assumed to be varying on scales slow compared with periods and wavelengths. The terms $v_{g 0}, v_{g s}$ $v_{g e}$ are relevant group velocities. The linear damping $\gamma$ is the sum of Landau damping $\omega_{i(\mathrm{LD})}$ and the effect of collisions $v_{\text {col }} / 2$ (zero in our Vlasov code case). The $\left|E_{e}\right|^{2}$ term in Eq. (4) reflects, for completeness, the effects of relativistic mismatch. ${ }^{11}$ (In fact, the relativistic detuning effect proved to be minor in the cases studied here, as was verified by removing the detuning term and examining the resulting differences.)

Often, the conservation of action density for equations such as Eqs. (4a) $-(4 c)$ is inferred to only within a multiplicative constant, after one sets $\partial / \partial x$ and damping equal to zero and performs the usual manipulation with complex conjugate variables and equations. Since we wish to compare with actual numerical results from the Euler-Vlasov code, constants are important. (All too often, theorists begin with the complex action amplitude equations and undefined coupling factors, which make it very difficult for many experimenters to relate the analysis to an experiment.) Here, we have begun with field quantities because we wish to be able to then go directly to the complex action forms, rather than to some quantity proportional to them. The most direct route is to define directly the complex action amplitudes $a_{(0, s, e)}$, such that the action density $S$ $\left(=a a^{*}\right)$ is given from the energy density $W$ (in SI units) by

$$
\begin{aligned}
S & =a a^{*}=\frac{W}{\omega}=\frac{\langle\text { Energy density }\rangle_{\omega}}{\omega} \\
& =\frac{1}{4 \omega}\left[\mu_{0}|H|^{2}+\epsilon_{0} E\left(\frac{\partial \omega \epsilon_{r}}{\partial \omega}\right) E^{*}\right] .
\end{aligned}
$$

[Here, $\epsilon_{r}$ is the transverse $(r=o, s)$ or longitudinal $(r=e)$ relative dimensionless dielectric coefficient, as the case may be, and $\epsilon_{0}, \mu_{0}$ are the usual SI vacuum quantities, while $E$ and $H$ are the appropriate electric and magnetic fields.] For our three waves, this gives the following:

$$
\begin{aligned}
& a_{0}=\left(\epsilon_{0} \omega_{0} / 2\right)^{1 / 2} A_{0}, \\
& a_{s}=\left(\epsilon_{0} \omega_{s} / 2\right)^{1 / 2} A_{s}, \\
& a_{e}=\left(\epsilon_{0} \omega_{e} / 2\right)^{1 / 2} \omega_{p}^{-1} E_{e} .
\end{aligned}
$$

[For the last result, for consistency, one must use the exact form from the electron fluid approximation $\epsilon$ $=1-\omega_{p}^{2} /\left(\omega_{e}^{2}-3 k_{e}^{2} v_{t h}^{2}\right)$ to get $W_{e}=(1 / 2) \epsilon_{0} E_{e} E_{e}^{*} \omega_{e}^{2} / \omega_{p}^{2}$. If one does not, the action conservation for zero energy loss will not emerge.]

The usual advantage of using the complex action density amplitudes is the evident symmetry of the coupling coefficients in the equations, a symmetry that was lacking in Eqs. $(4 a)-(4 c)$ : 
$\left(\frac{\partial}{\partial t}+v_{g 0} \frac{\partial}{\partial x}\right) a_{0}=-\Gamma a_{s} a_{e} e^{i \Delta \omega t}$,

$$
\left(\frac{\partial}{\partial t}+v_{g s} \frac{\partial}{\partial x}\right) a_{s}=\Gamma a_{0} a_{e}^{*} e^{-i \Delta \omega t},
$$

$$
\left(\frac{\partial}{\partial t}+\gamma+v_{g e} \frac{\partial}{\partial x}\right) a_{e}+i \frac{3}{4}\left(\frac{e \omega_{e}}{m c}\right)^{2} \frac{\left|a_{e}\right|^{2}}{\omega_{p}^{2} \epsilon_{0}} a_{e}=\Gamma a_{0} a_{s}^{*} e^{-i \Delta \omega t} .
$$

Here, we have defined

$$
\Gamma=(e / 2 m)\left[k_{e} \omega_{p} /\left(2 \epsilon_{0} \omega_{0} \omega_{s} \omega_{e}\right)^{1 / 2}\right] .
$$

When one can drop the $\partial / \partial x$ terms, as we can here for our spatially periodic case, one can obtain the usual action sums in the standard way:

$$
\begin{aligned}
& C_{s}=S_{0}+S_{s}=\left|a_{0}\right|^{2}+\left|a_{s}\right|^{2}=W_{0} / \omega_{0}+W_{s} / \omega_{s} \\
& =C_{s}(t=0) \text {, } \\
& C_{e}(t)=S_{0}+S_{e}=\left|a_{0}\right|^{2}+\left|a_{e}\right|^{2}=\frac{W_{0}}{\omega_{0}}+\frac{W_{e}}{\omega_{e}} \\
& =C_{e}(t=0)-\omega_{e}^{-1} \int_{0}^{t} d t \frac{d W_{e}}{d t} \text { (diss) } \\
& =C_{e}(t=0)-2 \gamma \omega_{e}^{-1} \int_{0}^{t} d t W_{e} .
\end{aligned}
$$

Note that any detuning (i.e., $\Delta \omega \neq 0$ ) does not directly affect the action conservation; the effect is indirect, limiting rather the extent to which action can be converted. These action density results are given in terms of (energy density/frequency) since it is easy to obtain squares of mode field amplitudes at any instant from the EVF code (since only instantaneous values are involved) and to apply the calculated eigenfrequencies to give the action densities from Eqs. (6a)-(6e).

In order to check the small signal behavior, we required the usual linear coupled mode eigenvalues. That is, with $d / d t \Rightarrow p$ and an initial pump $a_{0}$ and very small values for either or both of $a_{s}$ and $a_{e}$, i.e., $\left|a_{s}\right|^{2},\left|a_{e}\right|^{2}<\left|a_{0}\right|^{2}$, one has the usual coupled-mode dispersion relation from the determinant of the coefficients for the daughter waves [as given in Eq. (B5)]:

$$
\left(p+\gamma_{e}+i \Delta \omega / 2\right)\left(p+\gamma_{s}-i \Delta \omega / 2\right)-\Gamma^{2}\left|a_{0}\right|^{2}=0 .
$$

(Here, we have introduced a transverse wave damping $\gamma_{s}$ just to show the symmetry, it is really zero in our problem.) This has the usual quadratic solution pair for $p$ :

$$
\begin{aligned}
p= & -\left(\gamma_{e}+\gamma_{s}\right) / 2 \pm\left\{\Gamma^{2}\left|a_{0}\right|^{2}\right. \\
& \left.-\left[\Delta \omega-i\left(\gamma_{e}-\gamma_{s}\right)\right]^{2} / 4\right\}^{1 / 2},
\end{aligned}
$$

which, for nearly negligible damping, approximates to

$$
p \approx-\left(\gamma_{e}+\gamma_{s}\right) / 2 \pm\left[\Gamma^{2}\left|a_{0}\right|^{2}-(\Delta \omega / 2)^{2}\right]^{1 / 2} .
$$

For small damping and small mismatch, one can approximately separate real and imaginary parts explicitly:

$$
\begin{aligned}
p_{ \pm} \approx & -\frac{\gamma_{e}+\gamma_{s}}{2} \pm \Gamma\left|a_{0}\right|\left(1-\frac{1}{8} \frac{\Delta \omega^{2}-\left(\gamma_{e}-\gamma_{s}\right)^{2}}{\left(\Gamma^{2}\left|a_{0}\right|^{2}\right)}\right) \\
& \pm \frac{i}{4} \frac{\Delta \omega\left(\gamma_{e}-\gamma_{s}\right)}{\Gamma\left|a_{0}\right|} .
\end{aligned}
$$

From Eq. (11), for small damping and pump values below threshold, when the square-root term is largely imaginary, increasing the pump at first drives the real frequencies toward the matching condition to cancel the mismatch. Only when this has been done will the growth rate begin to increase. Above threshold, the reduced growth rate $\left[\Gamma^{2}\left|a_{0}\right|^{2}-(\Delta \omega / 2)^{2}\right]^{1 / 2}$, compared with that for negligible mismatch, reflects this initial pump "investment" required to force a match before being able to grow synchronously. In a periodic simulation with discrete $k$ modes, this phenomenon can be important in reducing growth if the $k$-mode combination frequency proves to have significant (mismatch) ${ }^{2}$ compared with the (basic growth term) ${ }^{2}$.

As will be seen (in Sec. IV A), the $\Gamma\left|a_{0}\right|$ growth term for our simulation conditions is $2.538 \times 10^{-2} \omega_{p}$ so the mismatch must be carefully determined to be significantly better than $0.01 \omega_{p}$. This requires including relativistic effects. The relativistic effect is most dramatic for Landau damping, whose damping exponent is no longer than usual,

$$
\frac{-\omega_{e}^{2}}{\left(2 k_{e}^{2} v_{t e}^{2}\right)}=\frac{-\beta_{e}^{2} k_{\mathrm{B}} T_{e}}{m c^{2}} \quad\left(\beta_{e} \equiv \frac{\omega_{e}}{k_{e} c}\right),
$$

but the correct relativistic Maxwellian form $-\left[\left(1-\beta_{e}^{2}\right]^{-1 / 2}-1\right) k_{\mathrm{B}} T_{e} / m c^{2}$. Ignoring relativity in Landau damping, apart from the exponent, we have

$$
\begin{aligned}
\frac{\gamma}{\omega_{p}}=\frac{\omega_{i(\mathrm{LD})}}{\omega_{p}}= & \left(\frac{\pi}{8}\right)^{1 / 2} \beta_{e}^{3 / 2} \sum_{s} f_{s}\left(\frac{511}{T_{(s) \mathrm{keV}}}\right)^{3 / 2} \\
& \times \exp \left(-\left(\gamma_{e}-1\right) \frac{511}{T_{(s) \mathrm{keV}}}\right),
\end{aligned}
$$

where we include the possibility of " $s$ " electron thermal components with fractional density $f_{s}\left(=n_{s} / n\right)$ and temperature (in keV) of $T_{(s) \mathrm{keV}}$ [ $\left.=511\left(k_{\mathrm{B}} T_{s} / m c^{2}\right)\right]$. [Recall $\gamma_{e}=\left(1-\beta_{e}^{2}\right)^{-1 / 2}, \beta_{e}=\omega_{e} / k_{e} c=\left(\omega_{e} / \omega_{p}\right)\left(\omega_{p} / k_{e} c\right)$.]

The electrostatic dispersion equation, relativistically correct to first order in $k_{\mathrm{B}} T / m c^{2}$, is also needed for the precision desired, namely

$$
\frac{\omega_{e}^{2}}{\omega_{p}^{2}} \approx 1+\sum_{s} \int_{s} \frac{T_{\mathrm{keV}(s)}}{511}\left(3 \frac{k_{e}^{2} c^{2}}{\omega_{p}^{2}}-\frac{5}{2}\right) .
$$

(The last term is the relativistic correction.) [This correction can be considered as reflecting the relativistic change in electron inertia for hot electrons, equivalent to including the second term in the asymptotic expansion for the relativistic normalization constant $K_{2}\left(m c^{2} / K T_{s}\right)$, where $K_{2}$ is the usual modified Bessel (McDonald) function of order 2.]

To be physically consistent with an exact relativistic simulation, one should use the relativistic correction on the electromagnetic dispersion as well, but, in the simulation 
we analyze here, the nonrelativistic transverse equation is used [Eq. (2)], so the usual transverse dispersion equation applies, namely

$$
\omega_{0, s}^{2} / \omega_{p}^{2}=1+k_{0, s}^{2} c^{2} / \omega_{p}^{2}
$$

[The effect would have been about $1 \%$ on $\left(\omega_{0}-\omega_{s}\right) / \omega_{p}$.]

We have now considered the eigenvalue equations and the details of the calculation of the plasma mode frequencies, etc., which are needed, but more is often required to analyze growth than the simple idea of drawing a straight line on a semilogarithmic plot and comparing the slope with the growth rate given by the simulation. When one wishes to examine growth in an initial value problem, the actual initial state needs to be considered in some detail. Since it does not seems to be given in the literature, a discussion of the initial value problem is presented in Appendix B. For instance, in the lossless case with no mismatch, a significant pump, and only one weak wave initially present, the action density for that daughter wave grows as $\left|a_{e}\right|^{2}(t=0) \cosh ^{2}\left(\Gamma a_{0} t\right)$, while the other grows as $\left|a_{0}\right|^{2}(t=0) \sinh ^{2}\left(\Gamma a_{0} t\right)$.

In our basic simulation, initial conditions were chosen with the pump and daughter wave sufficiently large so as to ensure that pump depletion, etc., would occur within a reasonable time $\left(\omega_{p} t \sim 180\right)$. However, this also meant that the nonlinear saturation effects became noticeable at a time so small $\left(\omega_{p} t \sim 40\right)$ that asymptotic clear straight line behavior could not be seen on a logarithmic plot. Hence it was preferable to use fits to the form of the squares of hyperbolic sines and cosines.

If our goal had been simply to look at initial growth, then with this noiseless EVF code, we could have used a much lower initial value for the perturbed plasma wave but, given that our principal goal was to examine the particle trapping behavior in the nonlinear regime, that would have been self-defeating. However, to prove the point about linear growth rate, another run was, in fact, made later, with a much lower initial level for the daughter wave, so that the exponential growth could indeed be checked over a longer scale, as discussed at the end of Sec. IV. Of course, in the same computational time, saturation onset due to pump depletion was then barely reached. This option may not be available in a PIC code, however, since the initial noise often is such that beginning at a sufficiently low plasma wave level may not even be possible without an enormous number of macroparticles.

\section{SIMULATION OF ONE-DIMENSIONAL SRS IN VLASOV PLASMA}

\section{A. Simulation parameters}

As mentioned above, to prepare for our causal simulations discussed elsewhere, ${ }^{8}$ we performed numerical simulations using periodic boundary conditions and an initial state consisting of a significant electromagnetic pump propagating in the positive $x$ direction and a propagating electrostatic wave of modest amplitude. As discussed at the end of Sec. II, the convenient wave vector choice is mode 2 for the plasma wave [i.e., $k_{e}=2(2 \pi / L)$, where $L$ is the length of the computation region] and mode 4 for the pump with the forward-scattered wave mode number $k_{s}$ being, therefore, $(4-2) 2 \pi / L$, i.e., mode 2 .

Our $x-p_{x}$ phase space grid discretization was set at $128 \times 512$ and our time step was taken to be $0.03 \omega_{p}$, while $p_{\max } / m c$ was chosen to be 6.5 . Since the velocities are normalized to $c$, and frequencies to $\omega_{p}$, while the electromagnetic pump mode wave number has been chosen to be $2 \pi / 4 L$, the choice of $k_{0}\left(=2 k_{s}, 2 k_{e}\right)$ in effect determines $L$ in terms of $c / \omega_{p}$. For our plasma temperatures (a twocomponent plasma was used), a good frequency match was obtained by choosing $k_{0} c / \omega_{p}=2.4$ (and so $\left.k_{s} c / \omega_{p}=1.2=k_{e} c / \omega_{p}\right)$. The periodic box length is then (4/ 2.4) $2 \pi c / \omega_{p}$, i.e., $(5 / 3) \lambda_{p}$, with the spatial grid spacing $1 / 128$ of this.

From the electromagnetic dispersion [Eq. (16)], one now has $\omega_{0} / \omega_{p}=2.6, \omega_{s} / \omega_{p}=1.56205$, giving a frequency difference of $\left(\omega_{0} / \omega_{s}\right) / \omega_{p}=1.03795$.

The initial unnormalized pump fields are thus

$$
\begin{aligned}
E_{y}(x, t=0) & =E_{0} \cos \left(2.4 \omega_{p} x / c\right) \\
B_{z}(x, t=0) & =E_{0}\left(\frac{k_{0}}{\omega_{0}}\right) \cos \left(\frac{2.4 \omega_{p} x}{c}\right) \\
& =0.92308\left(\frac{E_{p}}{c}\right) \cos \left(\frac{2.4 \omega_{p} x}{c}\right) .
\end{aligned}
$$

[Of course, $v_{p}$ is also initialized consistently, i.e., $v_{y} / c=(1 / 2.6)\left(e E_{0} / m \omega_{p} c\right) \cos \left(2.4 \omega_{p} x / c\right)$. If this is not done, one not only has some waves going in the wrong direction, but also has the time-stationary transverse spatial oscillatory current discussed by Mori ${ }^{12}$.]

For our simulation, we take our $\left(\omega_{p}, c\right)$-normalized pump electric field $e E_{0} / \omega_{p} m c$ to be 0.28 , which gives $u_{\text {osc }}=e E_{0} / \omega_{0} m c=0.28 / 2.6=0.108$. [For comparison with practical laser cases it is useful to recall that $\left(p_{\text {osc }} / m c\right)^{2}=u_{\text {osc }}^{2} \quad$ (often loosely called " $v_{\text {osc }}$-on- $c$ squared") $=8.488 \times 10^{-19} I \lambda_{0 \mu}^{2} \mathrm{~W} / \mathrm{cm}^{2}$, so this is equivalent to a pump of intensity $1.37 \times 10^{16} \lambda_{0 \mu}^{-2} \mathrm{~W} / \mathrm{cm}^{2}$.]

From Sec. II and the $\left(\omega_{p}^{-1}, c\right)$ (time, velocity) normalization, the action is normalized by division with $n_{0} m c^{2} / \omega_{p}$ so the normalized pump action amplitude is

$$
\begin{aligned}
\left(\frac{S_{0} \omega_{p}}{n m c^{2}}\right)^{1 / 2} & =\left(\frac{\epsilon_{0} \omega_{0} \omega_{p}}{2 n m c^{2}}\left|A_{0}\right|^{2}\right)^{1 / 2} \\
& =\left(\frac{\omega_{0}}{2 \omega_{p}}\right)^{1 / 2} \cdot \frac{e E_{0}}{\omega_{0} m c}=\left(\frac{\omega_{p}}{2 \omega_{0}}\right)^{1 / 2} \cdot \frac{e E_{0}}{\omega_{p} m c}
\end{aligned}
$$

i.e., an initial value of 0.1228 .

The $\left(\omega_{p}, c\right)$-normalized value of $\Gamma$ is, from Eq. (8),

$$
G=\left(\frac{n_{0} m c^{2}}{\omega_{p}^{3}}\right) \Gamma=\frac{k_{e} c}{\omega_{p}} \cdot\left(\frac{\omega_{p}^{3}}{8 \omega_{0} \omega_{s} \omega_{e}}\right)^{1 / 2}=0.20664 .
$$

The normalized ideal growth rate without mismatch or loss is thus $0.1228 \times 0.20664$, i.e., a growth rate of $2.5375 \times 10^{-2} \omega_{p}$. [Of course, this is the same as the usual unnormalized result $k_{e} v_{\text {osc }} \omega_{p} / 4\left(\omega_{s} \omega_{e}\right)^{1 / 2}$, obtained ${ }^{5,9}$ with other variables.] As remarked above, since growth rate 
without loss or mismatch is about $2.5 \%$ of $\omega_{p \text {, the mis- }}$ match must be well calculated to at least that accuracy. (As an example, the nonrelativistic approximation gave such a large mismatch that growth would not have been predicted at all, quite apart from the fact that the overestimated Landau damping would have been even more fatal.)

The plasma was chosen with two electron temperature components, ${ }^{13}$ the majority $(0.95)$ component with a 15 $\mathrm{keV}$ temperature and a minority component $(0.05)$ at 100 $\mathrm{keV}$. [The cold temperature was high enough for electron Landau damping to subdue the usually rapidly growing but here unwanted backward SRS instability (BRS), while the hot component was enough to ensure sufficient electrons to study electron acceleration by the forward SRS (FRS) interaction, which is relevant to beatwave acceleration, without any initial beam-plasma instability from electrons accelerated by BRS.] For the $k_{e} c / \omega_{p}$ value of 1.2 , Eq. (16) gives (with $f_{1}=0.95, T_{\mathrm{keV}(1)}=15, f_{2}=0.05$, $\left.T_{\mathrm{keV}(2)}=100\right)$ a value of $\omega_{e}^{2} / \omega_{p}^{2}=1.0086$, hence $\omega_{e} / \omega_{p}=1.03383$, so $\beta_{e}=0.86144$ and $\gamma_{e}=1.969$, $u_{e}=0.9844$. Thus we have a normalized mismatch of $\Delta \omega / \omega_{p}=\left(\omega_{s}+\omega_{e}-\omega_{0}\right) / \omega_{p}=-1.549 \times 10^{-2}$, which has only a slight effect on growth. (If relatively had not been included, the $\omega_{e} / \omega_{p}$ valuc would have been $4.46 \times 10^{-2}$ higher and the normalized mismatch would have been $+2.9 \times 10^{-2}$.) Substituting the value of $\beta_{e}$ and $\gamma_{e}$ in Eq. (22) gives $\gamma / \omega_{p}=\omega_{i}(\mathrm{LD}) / \omega_{p}=1.6387 \times 10^{-3}$, whose effect is also small. (Had the nonrelativistic form been used here, the damping would have been incorrectly calculated at $3.35 \times 10^{-2} \omega_{p}-70$ times too large.)

Now one can calculate the characteristic coupled mode frequencies, substituting the above values into the standard result given in Eq. (11):

$$
p_{ \pm} / \omega_{p}=\left\{\begin{array}{r}
2.448 \times 10^{-2}-i 6.833 \times 10^{-5} \\
-2.612 \times 10^{-2}+i 6.833 \times 10^{-5}
\end{array}\right.
$$

One essentially has the approximation of small loss and negligible mismatch [Eq. (12)]:

$$
p_{ \pm} / \omega_{p} \approx\left(-\gamma_{e} / 2 \pm \Gamma\left|a_{0}\right|\right) / \omega_{p}=\left\{\begin{array}{r}
2.455 \times 10^{-2} \\
-2.619 \times 10^{-2}
\end{array}\right.
$$

In order to trigger the FRS instability in the EVF code (which is essentially noiseless), the electron distribution function was initially set to give a forward-propagating longitudinal wave at the outset:

$$
\begin{aligned}
f\left(x, p_{x}, t=0\right)= & {\left[1+\epsilon \frac{v_{\mathrm{th}}}{2} \frac{k_{e} c}{\omega_{p}} \cos \left(\frac{k_{e} c}{\omega_{p}} \frac{\omega_{p} x}{c}\right)\right] } \\
& \times F\left[p_{x}-\epsilon m v_{\mathrm{th}} \cos \left(k_{e} x\right)\right] .
\end{aligned}
$$

Here, $F\left(p_{x}\right)$ is the two-temperature Maxwellian distribution discussed above and $v_{\text {th }}$ is the cold thermal velocity (i.e., $v_{\mathrm{th}}^{2}=k_{\mathrm{B}} T / \mathrm{m}$ ). We have a positive-going wavenormalized energy density of $\left\langle w_{e}\right\rangle / n_{0} m c^{2}=(\epsilon / 2)\left(v_{\mathrm{th}} / c\right)^{2}$. With $\left(v_{\text {th }} / c\right)^{2}=15 / 511$ (for $15 \mathrm{keV}$ temperature) and $\epsilon=2^{1 / 2} / 10$, this gives an initial normalized plasma wave density of $1.468 \times 10^{-4}$. The normalized action density is, of course, $\omega_{e} / \omega_{p}(1.03373)$ times smaller.

Now that the initial conditions have been suitably and carefully chosen, it remained only to run the EVF code and to look at the action densities resulting from the EVF plasma simulation of the FRS problem.

\section{B. Simulation results: Wave action}

The time behavior of the action densities from the EVF simulation for FRS are shown in Figs. 1(a) and 1(b) together with the relevant action sums: the electromagnctic pair $S_{0}, S_{s}$ and $C_{s}$ in Fig. 1(a), and the pump and (lossy) electrostatic actions $S_{0}, S_{e}$, and $C_{e}$ in Fig. $1(\mathrm{~b})$.

The general behavior is as expected from the canonical parametric oscillator decay model: nonlinear and decaying oscillatory transfer from pump to daughter and back again. As expected, the action sum $C_{s}$ for the undamped electromagnetic wave pair is well conserved (to within $1.5 \%$ ), while the pump-plus-plasma action sum $C_{e}$ generally decreases (with an exception noted below), also as one would expect, since it involves a plasma wave that can accelerate particles, and thus lose energy and action.

Even before any detailed comparison with theory, it is noteworthy here [Fig. 1(b)] that this plasma wave action loss $\left(\sim-d C_{e} / d t\right)$ behaves nonlinearly. It increases significantly more than expected as the plasma wave builds up to significant levels and then actually reverses slightly as the plasma wave decreases. [A linear damping term would, of course, mirror the plasma wave action variation $-d C_{e} / d t=2 \gamma S_{e}$ of Eq. (9).] The initial damping is nonetheless too small to slow the initial growth noticeably and so this action loss occurs as significant slackening of linear growth becomes evident at $\omega_{p} t$ values of about 50 . This is the effect of increased damping due to significant particle trapping. The slight increase in $C_{e}$ as the plasma wave decreases again is almost certainly a nonlinear version of negative electron Landau damping with trapped particles trying to sustain a decreasing electron plasma wave that is (so to speak) "trying" to rebuild the pump. Without looking at action conservation combinations, this phenomenon could not have been detected, since there would have been no way to isolate the changes in damping and plasma wave absorption from the time-varying pump-times-scatteredwave source term.

In a medium with truly entropic action loss (evident here as the initial less the current value of $C_{e}$ ), the lost energy is well mixed into the system. In this lossless Vlasov plasma, with a single plasma wave being responsible for the energy transfer to fast electrons, we hypothesized that the kinetic energy of all the electrons above the lower momentum boundary of the wave separatrix would account for the missing energy (and on division by the electron plasma wave frequency) for the action $C_{k}$ given to the electrons. In Fig. 1(c), where $C_{k}$ is given with the components of Fig. 1(b), one can see clearly that adding $C_{k}$ to the $C_{e}$ of Fig. 1 (b) indeed gives a total $C_{e T}$, which is well conserved.

It should be noted that this is possible only when the accelerating waves are well defined and distinct (as here). If more than one plasma wave was involved with overlap- 

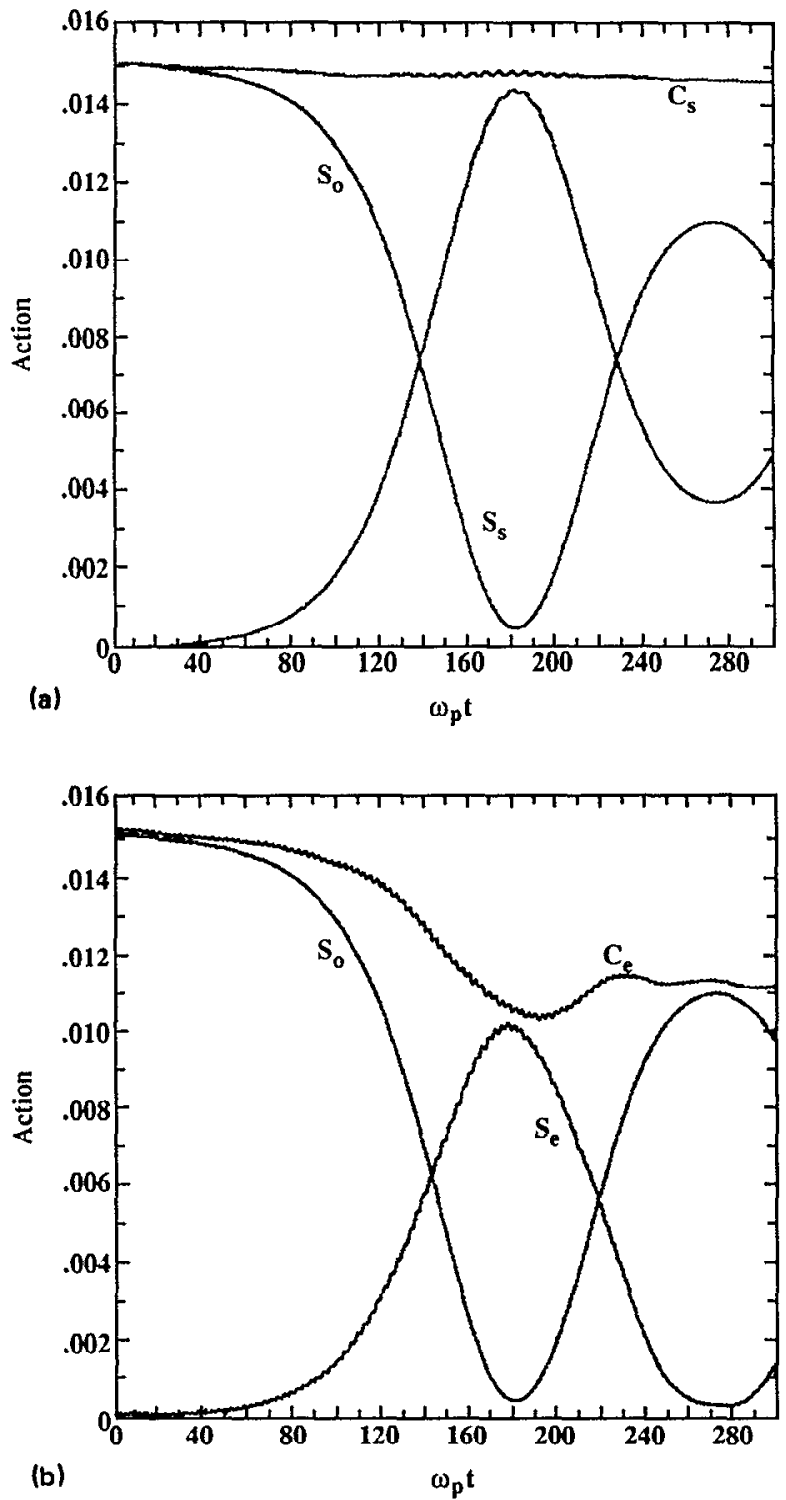

ping separatrices, one would expect Hamiltonian stochasticity from this resonance overlap. Even if the lowest separatrix could be well identified and the electron kinetic energy thus accounted for, one would not know to which wave one should ascribe a given region of the phase space distribution nor what frequency to use for division. In effect, one could say that, in this chaotic situation, the waves, so to speak, could only quarrel over the electrons without being able to establish a clear title of ownership.

Next, we decided to see if the three-oscillator model, with ad hoc damping to represent trapped electrons, could fit the simulation results of Fig. (1).

\section{THREE-OSCILLATOR APPROXIMATION FOR PERIODIC SRS}

The three-oscillator model of ideal parametric spatially uniform parametric decay is well known, but in this case it is the nonideal behavior that is of interest. The model consists of the set of equations (7a)-(7c), with spatial derivatives set to zero, and with normalized time $\tau=\omega_{p} t$, damping $D=\gamma / \omega_{p}$, and mismatch $\Delta \Omega=\Delta \omega / \omega_{p}$, normalized

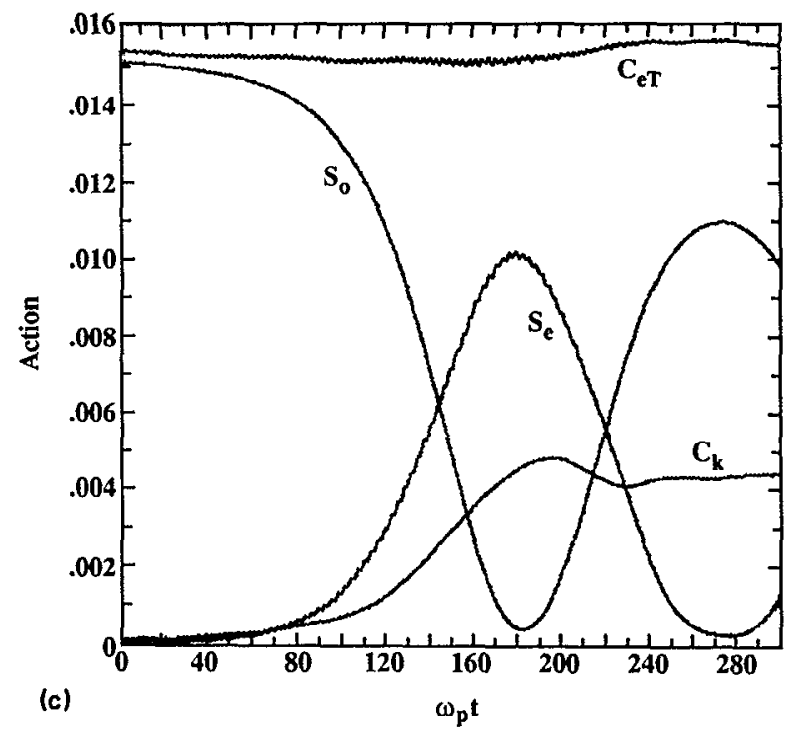

FIG. 1. From a spatially periodic initial-value Euler-Vlasov simulation of FRS: time evolution for the pump action $S_{0}$, scattered wave action $S_{s}$, and electron plasma wave action $S_{e}$ and various action sums $C_{s}\left(=S_{0}+S_{s}\right)$ and $C_{e}\left(=S_{0}+S_{e}\right)$. (a) Pump $S_{0}$, scattered FRS em wave $S_{s}$ and their action sum $C_{s}$ (b) Pump $S_{0}$, electron plasma wave $S_{e}$ and their action sum $C_{e^{\prime}}$ (c) As in (b), together with $C_{k}$ and $C_{e T}$, where $C_{k}$ accounts for particle accelerated above the lower separatrix (see Fig. 5) together with the total $C_{e T}\left(=C_{e}+C_{k}\right)$.

action $b_{0, s, \mathrm{e}}=a_{0, s, \mathrm{e}}\left(\omega_{p} / n_{0} m c^{2}\right)^{1 / 2}$, and the normalized coupling $G$ of Eq. (20):

$$
\begin{aligned}
& \frac{d b_{0}}{d \tau}=-G b_{s} b_{e} e^{i \Delta \Omega \tau}, \\
& \frac{d b_{s}}{d \tau}=G b_{0} b_{e}^{*} e^{-i \Delta \Omega \tau}, \\
& \frac{d b_{e}}{d \tau}+D b_{e}+i \frac{3}{4} \Omega_{e}^{2}\left|b_{e}\right|^{2}=G b_{0} b_{s}^{*} e^{-i \Delta \Omega \tau} .
\end{aligned}
$$

The value of $G$ is 0.20664 [Eq. (20)], while from the discussion between Eqs. (20) and (21), the Landau damping value for $D$ was found above to be $1.638 \times 10^{-3}$ and the calculated mismatch $-1.545 \times 10^{-2}$ with $\Omega_{e}=1.03373$. The initial value for $b_{0}$ was [from Eq. (19)] 0.1228, and for $b_{e}$ was $1.191 \times 10^{-2}$. (We had anticipated, however, that because of particle trapping and possible unanticipated frequency shift, a better average fit might be obtained by changing $D$ and/or $\Delta \Omega$ from their linear values. This had been undertaken before the action sum analysis of the EVF 

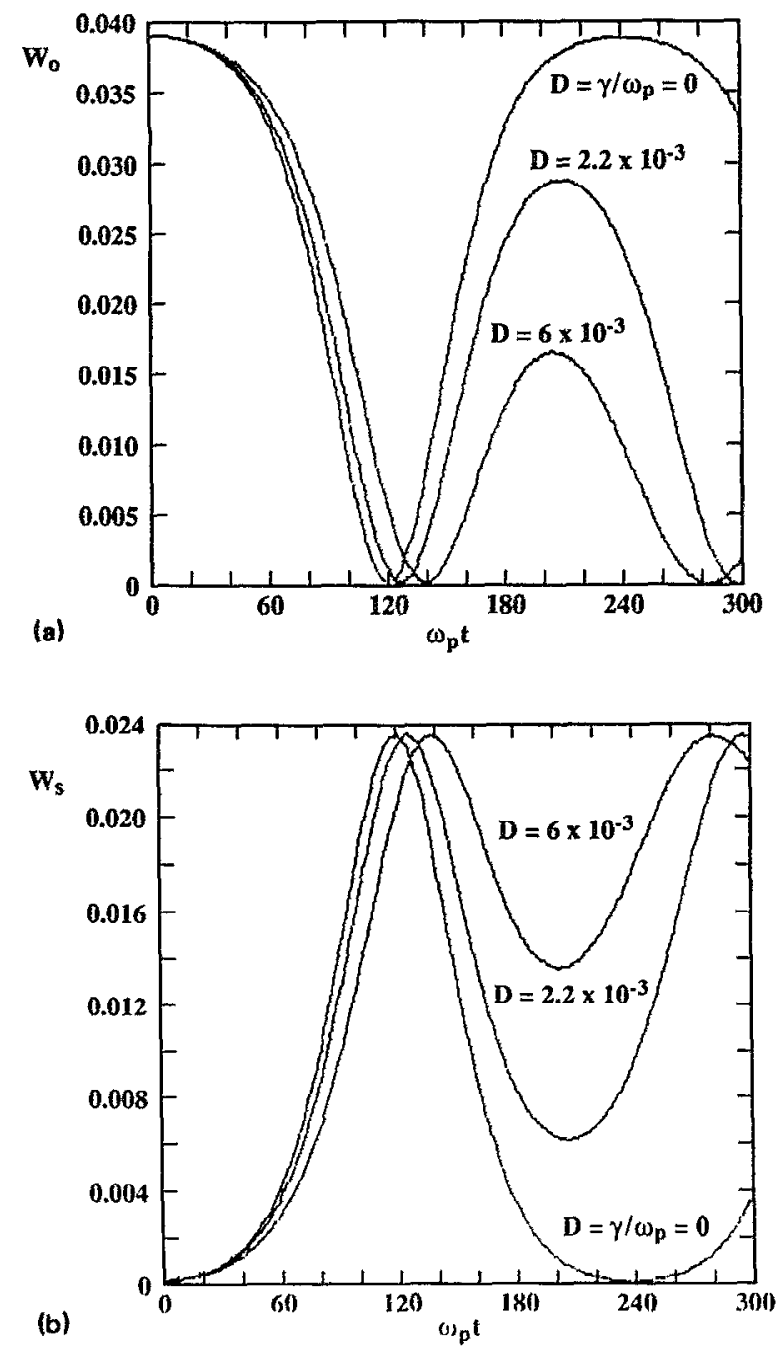

FIG, 2. Typical results from the three-oscillator coupling model: the effect of varying the plasma damping on the temporal behavior of (a) pump action, (b) scattered action.

simulation made it clear that the detailed time variation of the action loss could not be represented by linear ad hoc coefficients in the three-oscillator equations.)

Let us begin with fitting the linear damping. The energy results for the pump [Fig. 2(a)] and scattered SRS wave [Fig. 2(b)] of the three-oscillator model with no mismatch are shown for three assumed values of damping $D$ : $0,2.2 \times 10^{-3}$, and $6 \times 10^{-3}$, all of which affect only slightly the linear growth rate. With zero mismatch, on the first pump-depletion cycle (and indeed on all subsequent cycles), the pump depletes completely and the time of the pump depletion varies little with slight dissipation. The chief effect of changing the damping is to change (i) the value of the maximum value to which the pump can rebound and (ii) the maximum value of the plasma wave, both reflecting the integrated loss of action via plasma wave dissipation. Hence this integrated dissipation feature is used to choose $D$ (i.e., $\gamma / \omega_{p}$ ) for the best overall fit to the Vlasov simulation results. A reasonable fit was obtained with a $\gamma / \omega_{p}$ of about $2.2 \times 10^{-3}$. However, with that value,

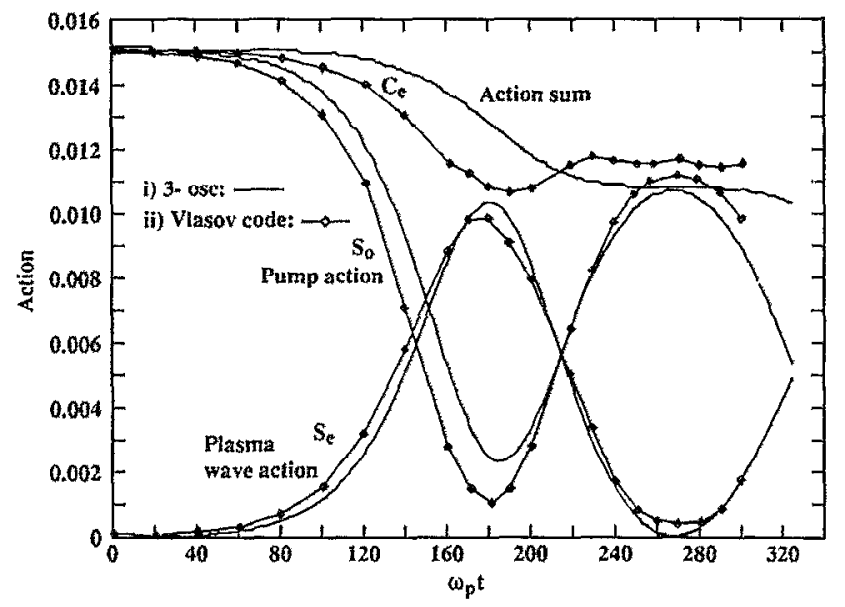

FIG. 3. Comparison of three-wave coupling (plain lines) and EVF (advanced by $\omega_{p} t=45$, see text) action results (lozenges). Temporal evolution of the pump and plasma actions $\left(S_{0}\right.$ and $\left.S_{e}\right)$ and their action sum $C_{e}$ for $\gamma_{e}=0.0025 \omega_{p}(1.53 \times$ Landau $)$ and $\Delta \omega=-0.025 \omega_{p}(1.62 \times$ linear calculation). Note the monotonic decrease of the action sum for the three-oscillator model contrasted with the more complex behavior due to nonlinear particle trapping effects.

the oscillator model depleted faster than the Vlasov simulation, reaching maximum depletion at an $\omega_{p} t$ value of about 45 earlier than the Euler-Vlasov code for reasons that are not clear.

The best fit for the nonlinear behavior was obtained by simply advancing these Euler-Vlasov results by an $\omega_{p} t$ value of 45 to compare them with the three-oscillator model. While it is only to be expected that there might be some sort of "synchronization time" before the asymptotic, slowly varying solution develops, the $\omega_{p} t$ value of 45 for such a time may seem large. In fact, this value is only seven cycles or so of plasma oscillation, a significant time on the scale of the slowly varying asymptotic solution. [That this delay is not a fundamental problem is indicated by the fact that, with a much smaller initial value for the Vlasov plasma wave, this "synchronization delay" was much reduced, to an $\omega_{p} t$ value of only 15 , or about 2.4 plasma oscillation cycles (see below).]

Next, we investigated the effect of changing the assumed three-oscillator mismatch, for relatively small mismatch and damping. Since there is only a slight effect on the initial growth, the mismatch is best diagnosed from the failure of complete pump depletion. A value for $\Delta \omega / \omega_{p}$ of about 0.013 to 0.025 gave a reasonable fit.

The best overall fit with the Euler-Vlasov results used $\gamma / \omega_{p}=2.5 \times 10^{-3}$ (1.53 $\times$ the relativistic Landau value) and $\Delta \omega / \omega_{p}$ of 0.025 ( $1.62 \times$ linear calculation) (with the Euler-Vlasov $\omega_{p} t$ values reduced by 45 ), as shown in Fig. 3.

In Fig. 3, we have also presented the action sums of Fig. 1(b) for comparison. With damping and mismatch close to the linear values, the three-oscillator model does a fairly good job (providing, as mentioned above, we apply the effective delay to the Vlasov results equal to an $\omega_{p} t$ value of 45). We repeat, however, that the EVF simulation action sum of pump and electrostatic wave actually shows 


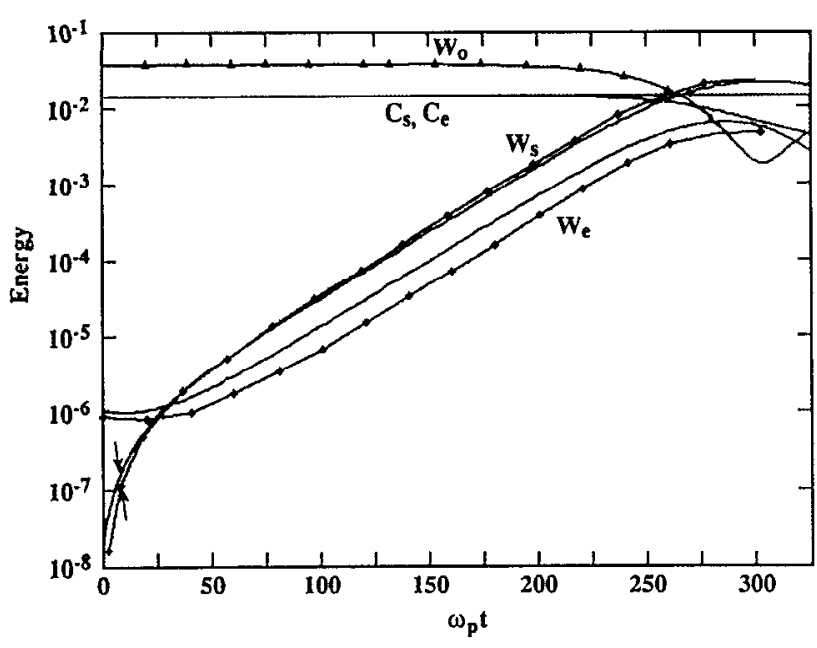

FIG. 4. These results are the energy equivalent (i.e., $W$ s instead of $S$ 's) of those in Fig. 3, except that (i) the initial plasma wave amplitude is only 0.134 times as large as that of Fig. 3, (ii) the Euler-Vlasov results are here advanced by only $\omega_{p} t$ value of 15 instead of 45 , and the results are shown on a logarithm scale so the exponential/cosh/sinh temporal behavior can be more clearly seen. The best-fit damping, etc., were here determined by fitting to the growth rate, particularly for the scattered wave (most easily measured in an experiment). The best-fit normalized damping now proved to be 0.01 ( 6.1 times the linear value), and even that gave a plasma wave peak cnergy that was $40 \%$ larger than the EVF simulation.

a slight rebound or regeneration instead of the series of downward-ramps-plus-flats expected from the linear damping, for which the action loss rate is strictly proportional to the electrostatic wave action. All this shows that, while the three-oscillator model can fit the behavior quite well, the plasma wave damping due to the trapping process cannot really be represented by a linear loss term; trapping dynamics must be considered.

As remarked above, in order to reach saturation and trapping phenomena in a reasonable time, the simulation was run with a sufficiently large initial value for the plasma wave so as to reach saturation in $\omega_{p} t$ values of 300 or so. This precluded a long-enough period of exponential growth of at least 2 decades to really check exponential growth with negligible pump depletion. In order to see this exponential phase more clearly, a further run was done with a much lower (by a factor of 0.134 ) initial plasma wave amplitude. These results, presented in Fig. 4, showed fairly good agreement with the three-oscillator model, but with a much lower value of apparent Euler-Vlasov delay ( $\omega_{p} t$ about 15) than for the simulation of Fig. 3, with the higher initial value for the plasma wave. Excellent agreement is obtained for exponential growth rates and relative amplitudes for the two daughter waves, but now the required linear damping factor for the integrated action loss is now much larger, 0.01 , which is 6.1 times the relativistic Landau value. The longer period of significant nonlinear trapping as the mode is growing from very low values can only be simulated with the increased value for linear damping.

From these two cases, it seems that the damping required for best performance for the three-oscillator model with the same strength pump and plasma conditions depends not only on the plasma conditions and perturbation wave number but also on the amplitude of the initial oscillation. Hence the best-fit ad hoc damping coefficient is therefore not readily predicted by linear calculations. While it might be useful to determine, via this sort of Manley-Rowe action-sum analysis, the time-varying effective relative plasma wave action loss rate for the simulation, to do so would be of little purpose without some glimmering of a numerical model for the trapping process. Clearly, the time derivative of the plasma wave energy is involved in some way, since for the same amplitude and for the first cycle at least, the action loss rate is much larger on the rising part of the plasma wave and much less (even becoming negative) on the falling part of the plasma wave.

Further progress will depend on improving our understanding of wave-particle dynamics in the presence of plasma waves that are seen in the wave or particle framc to be varying, all of which make up the next section.

\section{ACCELERATED PARTICLE DYNAMICS}

In an earlier paper $^{8}$ dealing with an open, causal system, we have shown that the EVF code is able to give strikingly clear pictures of the phase space dynamics, showing impressive correlation with simple orbit theory for steady waves, even though the waves are not steady, so that wave separatrices, strictly speaking, do not exist. In this section, we apply the orbit separatrix concept in more detail to our periodic EVF simulation of FRS and discuss some features of the complex structures of the electron phase space distribution function that are produced.

We recall that, from the separatrix orbit condition [Eq. (23) of the carlier paper ${ }^{8}$ ], one can readily solve the resulting quadratic equation for normalized separatrix momentum value $p_{x p}(x) / m c$ :

$p_{x p}(x) / m c=u_{\phi}[1+\Delta u(x)] \pm \gamma_{\phi}\left\{[1+\Delta u(x)]^{2}-1\right\}^{1 / 2}$,

where

$$
\Delta u(x)=-\left(e \gamma_{\phi} / m c^{2}\right)\left[\Phi_{0}-\Phi_{\mathrm{Lab}}(x)\right] .
$$

Here, $\Phi_{0}$ is the laboratory potential value at an X point, where $\Delta u$ is zero and the two separatrix values are the same. It is also useful to recall the plasma wave phase velocity quantities (denoted by a subscript $\phi$ ): $\beta_{\phi}=\omega_{e} / k_{e} \mathcal{c}$, $\gamma_{\phi}=\left(1-\beta_{\phi}^{2}\right)^{-1 / 2}, u_{\phi}=\beta_{\phi} \gamma_{\phi}=\left(\gamma_{\phi}^{2}-1\right)^{1 / 2}$.

For our sinusoidal waves, we have

$$
\Delta u=\Delta u_{0}\left\{1-\sin ^{2}\left[0.5 k_{e}\left(x-x_{0}\right)\right]\right\} \text {. }
$$

In Fig. 5, we present the separatrices calculated in this way from the simulation electrostatic field superimposed on the very accurate phase space representation afforded by the EVF code. Here, gray shading has been used to indicate the relative values of normalized phase space density between $5 \times 10^{-3}$ and $10^{-4}$ (with black for higher values and white for lower values).

It is the inherent phase space resolution of the EVF code that allows the prescntation of such detailed structures in phase space, which in the more common PIC 



FIG. 5. Phase space ( $p_{x} / m c$ vs $\left.x \omega_{p} / c\right)$ electron distribution function gray density plots (gray for $10^{-4}<f<5 \times 10^{-3}$, black for $f>5 \times 10^{-3}$, and white for $f<10^{-4}$ ) for various values of $\omega_{p} t$. The separatrices obtained from Eqs. (24) and (25) are also drawn. 

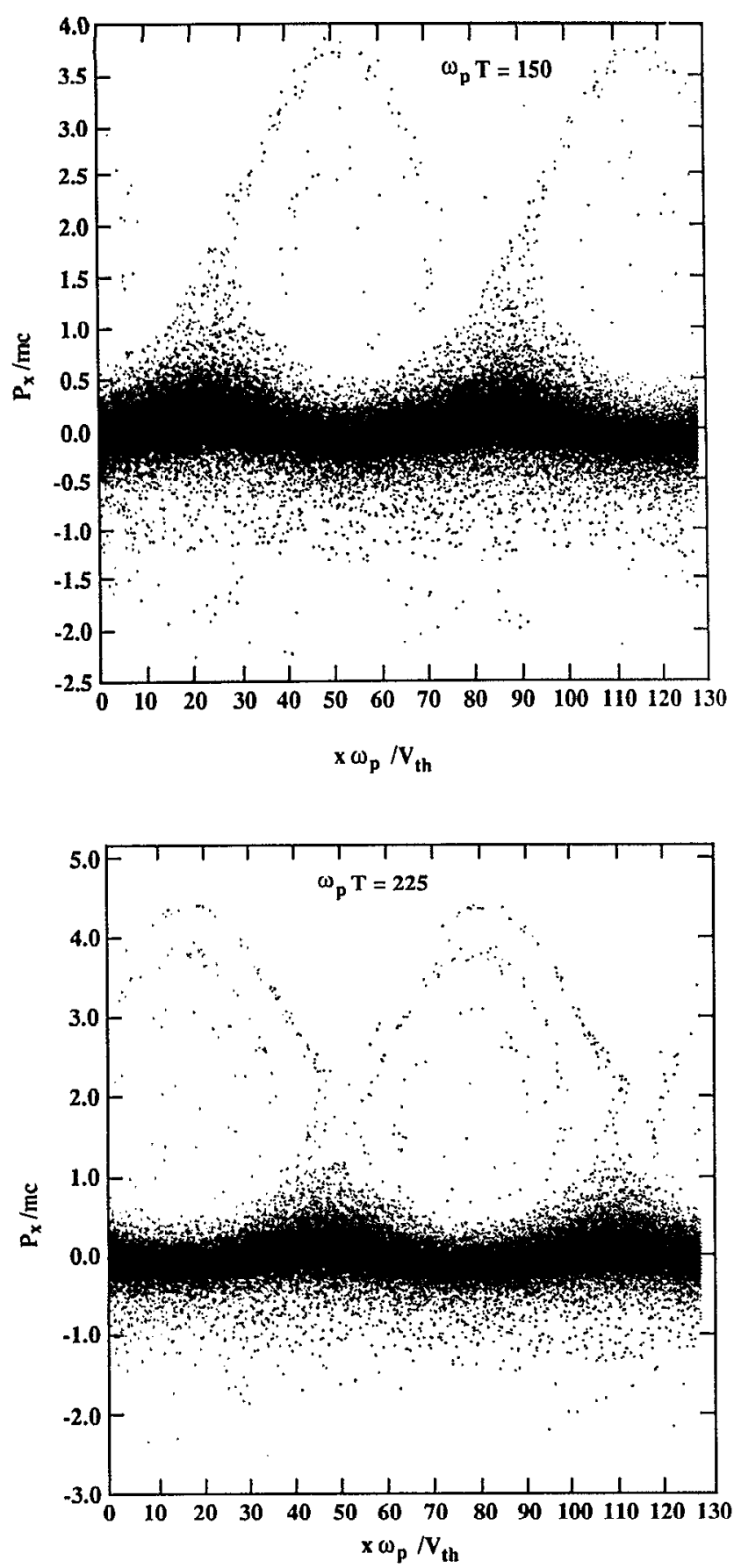

FIG. 6. For general comparison with Fig. 6, PIC electron positions in phase space at various times are shown as obtained by Brunel (unpublished).

codes are very coarsely represented by a small number of particles. Some unpublished work with Brunel of the Canadian National Research Council (using his PIC code) has indeed verified that these trapping spiral structures associated with a growing plasma wave coincide well with the location of accelerated PIC particles for equivalent runs. Two examples of these PIC results are shown in Fig. 6 . Since the phase space localized physics behavior in the two types of codes is very similar, it is clear that the essential difficulty in using a PIC code to obtain the kind of phase space detail available with the EVF code lies in arranging to have enough microparticles in phase space regions of interest without having to provide enormous numbers of these microparticles in uninteresting regions of phase space.

In the two-wavelength $(m=2)$ structure for the plasma wave in Fig. 5, one can clearly see the striking agreement between the separatrices calculated from the EVF code electric field and the phase space trapping limits for growing waves. The simple spiral structure inside the trapping limit in the early frames clearly reflects the monotonic increase in wave energy; the particles in the interior spirals, which are now very deeply trapped near the wave momentum value $m c u_{\phi}$, are distributed in this spiral form because they were trapped at earlier times when the wave was relatively small. (Another way to understand the spiral structure is to recall the duality that exists under time renormalization between the phase space behavior in a temporally growing oscillator $[\sim \exp (\alpha t)]$ without losses and the behavior of a system with an equivalent loss $[\sim \exp (-\alpha t)]$, when the phase space trajectory is a spiral to the local O-point attractor.) It is only the relatively recently and marginally trapped particles that can have momenta and energies given by the larger of the two values of Eq. (25), values that differ substantially from the value $u_{\phi}$ characteristic of the wave phase velocity. At the frame for $\omega_{p} t=180$, the amplitude of the plasma wave is near its maximum, with a maximum separatrix momentum (near $\omega_{p} x / c$ values of 2 and 7 ) of 5.0 times $m c$, and a local significant phase space density between momentum values of 4.7 and 5.1 times $m c$.

After the plasma wave maximum is reached, the plasma wave and the SRS wave begin to decrease as they recombine to reconstitute the pump. The marginally trapped particles now begin to detrap, as evidenced by the fact that they are now outside the wave separatrix boundaries (i.e., on the far side for each separatrix from the wave momentum value). This detrapping, which can be easily foreseen only by reference to the calculated separatrices, becomes manifest as the particles approach the separatrix $\mathrm{X}$ points. Instead of spiraling smoothly around the local $\mathrm{O}$ point as before when the wave was growing, the phase space arc near the $\mathrm{X}$ point develops a significant fold as the $\mathrm{X}$ point is approached from above (see $\omega_{p} t$ values of 195 and 210). Following this, the fold then rides up over the next separatrix hump as shown in the frames for $\omega_{p} t$ values of 225 and 240 . In this way, one sees explicitly by this folding process how the phase space distribution function continuity and the Liouville theorem are still satisfied as detrapping occurs. As the plasma wave decreases still further, the phase space evolution becomes that of simple relativistic convection (i.e., limited by the velocity of light).

Between $\omega_{p} t$ values of 270 and 285, the second decay cycle of the pump begins and with it the second rise of the plasma wave. The detrapped particles are now beginning to be retrapped. In frame 285 , the shallow phase space $V$ structures above and to the left of the $\mathrm{X}$ points are an early indication of this retrapping. By frame 300 , these shallow 
TABLE I. Electrostatic potential: Action estimates and code results.

\begin{tabular}{|c|c|c|c|c|c|c|c|}
\hline $\begin{array}{l}\text { EVF } \\
\text { simulation }\end{array}$ & $n_{0} / n_{c}$ & $\begin{array}{c}T \\
(\mathrm{keV})\end{array}$ & $\omega_{0} / \omega_{p}$ & $v_{\phi} / c$ & $v_{\mathrm{osc}} / c$ & $\begin{array}{c}e \phi_{\text {lab }} \\
m c^{2} \\
\text { theoretical } \\
\text { calculation }\end{array}$ & $\begin{array}{c}e \phi_{1 \mathrm{lab}} \\
m c^{2} \\
\text { code }\end{array}$ \\
\hline FRS I & $15 \%$ & 15.0 & 2.60 & 0.88 & 0.108 & 0.281 & 0.2080 \\
\hline FRS II & $15 \%$ & 15.0 & 2.60 & 0.88 & 0.123 & 0.321 & 0.2380 \\
\hline BRS & $18 \%$ & 3.3 & 2.34 & 0.38 & 0.120 & 0.126 & 0.0886 \\
\hline
\end{tabular}

$\mathrm{V}$ structures have become much deeper and the retrapping process is well advanced. (Of course, there are also similar phase space distribution function details involved with the lower separatrix, but these are much more difficult to see because of the large number of particles below the separatrix whose numbers effectively overwhelm these details of detrapping of a modest number of particles to lower momenta.)

It seems quite plausible that only a few such cycles would suffice to produce a distribution function that would change little under further cycles of trapping, detrapping, and retrapping and quite likely on a scale far more rapid than any beam-plasma instability-at least for low beam densities. Usual discussions of the stability of energetic tails consider either spatially uniform cases (with no spatial modulation for which the criteria are well known for electrostatic instability) or (more rarely) the sideband instability. It is evident that the stability criteria for these modulated but rather complex phase space distribution structures are probably well beyond the reach of present analysis. In our case, these phase space distribution function features in this problem do not exist on their own but in the presence of the waves with which they interact. However, in the causal problem, ${ }^{8}$ the electrons can outrun the region where they are generated, so the long-term stability of these beamlike structures on their own then becomes an issue to be addressed.

To understand particle-trapping dynamics, it is useful to estimate, via the Manley-Rowe relations, the maximum clectrostatic potential that would be obtained if the pump action were to be totally converted to daughter wave action. This is often what is done to discuss laser interaction experiments that produce significant quantities of accelerated electrons.

In the units we use here, the Manley-Rowe result, equivalent to

$$
\left(\frac{k_{e} \phi_{\mathrm{Lab}}}{m c \omega_{p}}\right)^{2} \omega_{e}=\left(\frac{v_{\mathrm{osc}}}{c}\right)^{2} \omega_{0}
$$

gives

$$
\frac{e \phi_{\mathrm{lab}}}{m c^{2}}=\left(\frac{\omega_{0}}{\omega_{e}}\right)^{1 / 2} \cdot \frac{\omega_{p}}{\omega_{e}} \cdot \frac{v_{\phi} v_{\mathrm{osc}}}{c^{2}},
$$

where $v_{\phi}=\omega_{e} / k_{e}$ is the linear phase velocity and $v_{\text {osc }}=e E_{0} / m \omega_{0}$ is the pump "quiver velocity" $p_{\text {osc }} / m c$. The results are shown in Table I [FRS I for the forward Raman scatter (FRS) result just discussed], together with two other simulations, namely: FRS II; the only change con- cerns $v_{\text {ose }}$, which is now $0.123 c$, and BRS; this is a backward (B) scattering, already published. ${ }^{7}$

Table I also lists the different parameters of these three simulations and provides a useful comparison between the theoretical value of the potential $\mathrm{Eq}$. (25) at saturation and the code value at the first plasma wave maximum.

As one might expect, theoretical calculation invoking the complete action conservation is a significant overestimate (by about $40 \%$ in all cases) for the final potential. To date, we have no easy a priori recipe for determining this shortfall.

In the case of FRS I, where we have done a careful accounting of the action and found (in Fig. 5) that 0.0097 / $0.0152(=0.6381)$ of the maximum plasma wave action to be recovered, we would expect the ratio of the actual-toideal potential to be the square root of this, namely 0.8 , but the ratio proves to be only 0.74 , leaving a still unexplained shortfall of $7.3 \%$. While the accounting seems not as precise as one could hope, we can say that the overestimate of plasma wave intensity via the Manley-Rowe relation is significant, even for comparatively small damping rates that affect the growth rate only slightly. The difference is indeed accounted for mostly by the transfer of plasma wave energy to the particles. Because the action transfer takes place relatively slowly, even small loss rates can accumulate significant action loss, resulting in a significant limit to the plasma wave energy, well below the ideal value for zero loss.

The failure of lossless theory to provide more than an upper bound is not surprising and indicates that the electron plasma wave amplitude cannot be inferred by pump depletion alone. However, one can say the following. Quick and violent wave growth will lead to higher maximum wave energy and higher energy for the most energetic particles, while slower and less violent wave growth will lead to lower wave energy and accelerated particle momenta much closer to the wave phase momenta $m c u_{\phi}$. Hence, to predict the distribution of accelerated particles with any accuracy, one must must know a great deal about the details and time scale of the nonlinear acceleration process. One can still say the following, however. Assuming a case where the plasma wave conversion process will go to significant levels, if high particle energies are desired (perhaps for an accelerator), rapid growth and intense pumping is to be encouraged. On the other hand, if high particle energies are not desired (as in laser fusion), gentle growth may well be preferred at low phase velocities.

\section{SUMMARY AND CONCLUSIONS}

Detailed phase space simulation results were obtained using a $1 \frac{1}{2}-\mathrm{D}$ Euler-Vlasov fluid (EVF) code for the spatially periodic, initial-value parametric instability problem of forward stimulated scattering by the plasma wave Raman process (FRS). Action conservation was satisfactorily verified for the two significant electromagnetic waves. We were also able to account very well for the plasma wave action loss by using the very detailed phase space diagnostics afforded by the Euler-Vlasov code together with plasma wave separatrices to allow classification of particle 
energy and action transfer from the plasma wave. The behavior of the system could be modeled in an average way by the standard coupled-mode equation with an appropriately chosen $a d$ hoc damping term. More detailed comparison showed that nonlinear damping effects were not negligible, depended on wave dynamics, and that some energy could be recovered by trapped-particle oscillation giving, in effect, limited negative Landau damping at times. Even though the damping effects duc to plasma particle acceleration were too small to affect wave growth significantly, they were important in limiting plasma wave amplitudes to values well below the collisionless maximum action transfer Manley-Rowe estimate. In cases where the plasma wave has other than a simple history of monotonic decay, the plasma particle distribution function in the region of the plasma wave phase velocity develops a complex structure of folds and streaks in phase space. It is clear that to describe the distribution of accelerated particle momenta and energy will require very detailed analysis of the kinetics and time history of the plasma wave evolution. Finally, obtaining these insights into this detailed behavior was only possible with an EVF code, which can provide a great deal of resolution in phase space.

This work on the spatially periodic case is intended as preparation for the application of similar analysis techniques to the simulations ${ }^{8}$ of the more realistic causal case where spatial periodicity no longer applies. In this latler case, one cannot use either time or space alone but must consider action conservation along the various wave characteristics. This work is now in hand.

\section{ACKNOWLEDGMENTS}

Our thanks are due to François Brunel for the simulation results of Fig. 6 .

The authors arc indebted to the Centre de Calcul Vectoriel pour la Recherche (École Polytechnique, Palaiseau, France) for computer time allocation on the Cray-2 computer. P. Bertrand and A. Ghizzo acknowledge gratefully the hospitality of the Centre Canadien de Fusion Magnétique and partial financial support of France-Québec during this work. The Centre Canadien de Fusion Magnétique is financed by Atomic Energy of Canada Limited, HydroQuébec and the Institut National de la Recherche Scientifique. T. W. Johnston's support is due to the Ministère de l'Education du Québec and to the National Science and Engineering Research Council of Canada. P. Bertrand and M. Feix acknowledge the partial financial support of the French Direction des recherches, études et techniques (Contract No. 88-1492).

\section{APPENDIX A: DERIVATION OF ONE-DIMENSIONAL SRS-COUPLED MODE EQUATIONS}

We wished to obtain coupled results in the form of action in order to use the action conservation results, but found the standard derivations not to be very convenient for this purpose. In this appendix, therefore, we provide a derivation of the couplcd-mode equations using field variables (rather than, say, density or current), since the en- ergy and action densities are then much easier to obtain using standard results with electric and magnetic fields [Eq. (5) in this paper]. For generality, we postpone until as late as possible in this derivation the assumption of harmonic time-space dependence and a slowly varying envelope.

We begin with the longitudinal component of the nonrelativistic electron momentum equation for the usual electron fluid:

$$
\frac{d m v_{x}}{d t}=-e\left(E_{x}+v_{y} B_{z}\right)-3 \frac{m v_{\mathrm{th}}^{2}}{n_{0}} \frac{\partial n_{e}}{\partial x} .
$$

(Linear damping is ignored here since it can easily be put in at the end.) Because we are only concerned here with transverse wave scattering involving the electron plasma waves, $n_{e}$ in the denominator is approximated by $n_{0}$ the unperturbed density. From the spatial derivative of the Poisson equation with immobile ions, we have

$$
\frac{\partial n_{e}}{\partial x}=\frac{-\epsilon_{0}}{e} \frac{\partial^{2} E_{x}}{\partial x^{2}} .
$$

Also, from the electron number density equation and the Poisson equation,

$$
\frac{\partial v_{x}}{\partial x}=-\frac{1}{n_{0}} \frac{\partial n_{e}}{\partial t}=\frac{\epsilon_{0}}{n_{0} e} \frac{\partial^{2} E_{x}}{\partial x \partial t} .
$$

Again, for the reason given above, $n_{e}$ is again approximated by $n_{0}$ in the denominator and the term $\left(v_{x} / n_{e}\right)\left(\partial n_{e} / \partial x\right)$ has been dropped. One can integrate Eq. (A3) on $x$ and then take the time derivative to infer

$$
\frac{\partial v_{x}}{\partial t}=\frac{\epsilon_{0}}{n_{e} e} \frac{\partial^{2} E_{x}}{\partial t^{2}} .
$$

Combining these results gives the electric field version of the plasma oscillation equation, but here including the $\mathbf{v} \times \mathbf{B}$ force term as well, namely

$$
\frac{\partial^{2} E_{x}}{\partial t^{2}}+\omega_{p}^{2} E_{x}-3 v_{\mathrm{th}}^{2} \frac{\partial^{2} E_{x}}{\partial x^{2}}=-\omega_{p}^{2} v_{y} B_{z}
$$

With the vector potential $A_{y}$ for $x$-directed electromagnetic radiation, the electric and magnetic fields in the usual Coulomb gauge are

$$
\begin{aligned}
& E_{y}=-\frac{\partial A_{y}}{\partial t}, \\
& B_{z}=\frac{\partial A_{y}}{\partial x} .
\end{aligned}
$$

In the nonrelativistic approximation $(p \approx m v)$, instead of the usual $p_{y}=e A_{y}$ for electrons we have

$$
v_{y}=e A_{y} / m \text {. }
$$

This value can now be used in Eq. (A5) together with Eq. (A6b) to give the field form of the plasma wave equation with a nonlinear electromagnetic field source term (essentially the ponderomotive force when appropriately time analyzed): 


$$
\frac{\partial^{2} E_{x}}{\partial t^{2}}+\omega_{p}^{2} E_{x}-3 v_{\mathrm{th}}^{2} \frac{\partial^{2} E_{x}}{\partial x^{2}}=-\omega_{p}^{2} \frac{e}{m} A_{y} \frac{\partial A_{y}}{\partial x} .
$$

Now, in the usual vector potential wave equation with the nonlinear current $J_{y} / \epsilon_{0}$ given by $-\left(n-n_{0}\right) e A_{y} / m \epsilon_{0}$, the transverse wave equation for $A_{y}$ becomes

$$
\frac{\partial^{2} A_{y}}{\partial t^{2}}-c^{2} \frac{\partial^{2} A_{y}}{\partial x^{2}}+\omega_{p}^{2} A_{y}=\frac{e A_{y}}{m} \frac{\partial E_{x}}{\partial x} .
$$

Now, we will apply the coupled-mode formalism to Eqs. (A8) and (A9) by making use of the usual assumption of a slowly varying envelope for each wave. Explicitly, this defines the complex amplitudes, first for the transverse waves,

$$
\begin{aligned}
A_{y}= & {\left[0.5 A_{0} \exp \left(-i \omega_{0} t+i k_{0} x\right)+\text { c.c. }\right] } \\
& +\left[0.5 A_{s} \exp \left(-i \omega_{s} t+i k_{s} x\right)+\text { c.c. }\right],
\end{aligned}
$$

and then for the electrostatic electron plasma wave,

$$
E_{x}=\left[0.5 E_{e} \exp \left(-i \omega_{e} t+i k_{e} x\right)+c_{. c} .\right],
$$

where the complex amplitudes $A_{0}, A_{s}$ and $E_{e}$ are assumed to be only slowly varying. Applying this to Eq. (A9) for the pump $\left(\omega_{0}, k_{0}\right)$ and using the standard cancellation due the zeroth-order dispersion relation leaves

$$
-i 2 \omega_{0}\left(\frac{\partial}{\partial t}+v_{g 0} \frac{\partial}{\partial x}\right) \frac{A_{0}}{2}=\frac{e}{m}\left(\frac{A_{s}}{2}\right)\left(\frac{i k_{e} E_{e}}{2}\right) e^{i \Delta \omega t}
$$

or, in the final form,

$$
\left(\frac{\partial}{\partial t}+v_{g 0} \frac{\partial}{\partial x}\right) A_{0}=-\frac{e}{4 m} \frac{k_{e}}{\omega_{0}} A_{s} E_{e} e^{i \Delta \omega t} .
$$

Here, $v_{g 0}\left(=c^{2} k_{0} / \omega_{0}\right)$ is the transverse wave group velocity. The procedure is similar for $A_{s}$, except that, as well as the obvious exchange of 0 and $s$ subscripts, one also has on the right, for resonance, the complex conjugate of $\left[i k_{e} E_{e} \exp (i \Delta \omega t)\right]$, so the result for $A_{s}$ differs in those important aspects from that of $A_{0}$ :

$$
\left(\frac{\partial}{\partial t}+v_{g s} \frac{\partial}{\partial x}\right) A_{s}=+\frac{e}{4 m} \frac{k_{e}}{\omega_{s}} A_{0} E_{g}^{*} e^{-i \Delta \omega t} .
$$

In a similar way, with the electron plasma wave group velocity $v_{g e}\left(=3 v_{\mathrm{th}}^{2} k_{e} / \omega_{e}\right)$, one obtains an equivalent equation for $E_{e}$ (with $k_{0}-k_{s}=k_{e}$ ):

$$
\begin{aligned}
{\left[\frac{\partial}{\partial t}\right.} & \left.+\gamma+v_{g s} \frac{\partial}{\partial x}+\left.i \frac{3}{8}\left(\frac{e}{m c}\right)^{2} \frac{\omega_{e}^{3}}{\omega_{p}^{4}}\left|E_{e}\right|\right|^{2}\right] E_{e} \\
& =-\frac{\omega_{p}^{2}}{-i \omega_{e}} \frac{e}{m}\left[\frac{A_{0}}{2}\left(\frac{i k_{s} A_{s}}{2}\right) *+\frac{A_{s}^{*}}{2}\left(\frac{i k_{0} A_{0}}{2}\right)\right] \\
& =\frac{e}{4 m} \frac{k_{e}}{\omega_{e}} \omega_{p}^{2} A_{0} A_{s}^{*} e^{-i \Delta \omega t} .
\end{aligned}
$$

In the last equation, we have introduced directly for completeness the damping $\gamma\left[=\omega_{i}\right.$ (Landau damping) $+v(\operatorname{coll}) / 2]$ as well as the correction ${ }^{11}$ for detuning due to relativistic electron inertia modification from the longitudinal oscillation.
Equations (A12b), (A13), and (A14) are equations $(4 a)-(4 c)$ of the paper.

\section{APPENDIX B: CHARACTERISTIC WAVE BEHAVIOR FOR UNSTABLE PARAMETRIC COUPLED MODES}

While much has been published on how to obtain the eigenvalues (growth rates, etc.) from parametric instability dispersion relations for uniform systems, the more detailed consideration of the temporal behavior for the coupled modes has been scarcely mentioned in the literature. The presentation of this mode behavior is the subject of this appendix.

The complex action amplitude coupled mode behavior for spatially uniform modes (so $\partial / \partial x \rightarrow 0$ ) is governed by the following pair of coupled ordinary differential equations:

$$
\begin{aligned}
& \frac{d a_{s}}{d t}+v_{s} a_{s}=\Gamma a_{0} a_{e}^{*} \exp (-i \Delta \omega t), \\
& \frac{d a_{e}^{*}}{d t}+v_{e} a_{e}^{*}=\Gamma a_{0}^{*} a_{s} \exp (+i \Delta \omega t) .
\end{aligned}
$$

The coupling coefficient $\Gamma$ and, here, the pump amplitude $a_{0}$ are taken to be constant as we are now analyzing the linear approximation of negligible pump depletion.

While one could attack the system formally with Laplace transform analysis, it is more physically transparent to consider the two pairs of coupled modes which, at $t=0$, are such that one of the component modes is at an arbitrary nonzero value and the value of the other is zero. These two coupled mode pairs are the useful eigenmode combinations that make it easy to analyze any initial state. Notice that the equations have a simple symmetry; taking the complex conjugate and then exchanging the labels " $e$ " and " $s$ " gives the same equations again. Hence we need only develop one of these modes explicitly, obtaining the other by symmetry. Since we set the scattered mode $a_{s}$ initially equal to zero in this paper for our simulations and in the three-oscillator model, this is the case we chose to analyze explicitly.

The assumption or ansatz we use for the solutions are the following:

$$
\begin{aligned}
a_{s}(t)= & a_{s+} \exp \left[\left(p_{+}-i \Delta \omega / 2\right) t\right]+a_{s-} \exp \left[\left(p_{-}\right.\right. \\
& -i \Delta \omega / 2) t], \\
a_{e}^{*}(t)= & a_{e+}^{*} \exp \left[\left(p_{+}+i \Delta \omega / 2\right) t\right]+a_{e-}^{*} \exp \left[\left(p_{-}\right.\right. \\
& +i \Delta \omega / 2) t] .
\end{aligned}
$$

The values $p_{+}, p_{-}$are the solutions to the standard eigenvalue equation obtained by setting the determinant of the equation pair (B1) and (B2) equal to zero, namely,

$$
\left(p+v_{s}-i \Delta \omega / 2\right)\left(p+v_{e}+i \Delta \omega / 2\right)-\Gamma^{2}\left|a_{0}\right|^{2}=0 .
$$

The solution to this quadratic gives $p_{+}, p_{-}$ 


$$
\begin{aligned}
p_{ \pm} & =-\frac{v_{e}+v_{s}}{2} \pm \sqrt{\Gamma^{2}\left|a_{0}\right|^{2}+\left(\frac{v_{e}-v_{s}+i \Delta \omega}{2}\right)^{2}} \\
& \equiv-\frac{s}{2} \pm d .
\end{aligned}
$$

Now in order to have $a_{s}(t=0)$ equal to zero, we must have $a_{s+}=-a_{s-} \equiv a / 2$, so we have, for $a_{s}(t)$,

$$
a_{s}=a \exp \left[\left(\frac{-s-i \Delta \omega}{2}\right) t\right] \sinh (d t) \text {. }
$$

The time-asymptotic behavior corresponding to $\exp \left(p_{+} t-i \Delta \omega t / 2\right)$ is clearly evident, as is the fact that $a_{s}$ is zero at $t=0$. The link to the initial value for $a_{e}^{*}$ is also easily established from Eq. (B1) at $t=0$. At $t=0$, from Eq. (B7), we see that the value of time derivative of $a_{s}$ is simply $a d$ and so we can solve Eq. (B1) for $a$ at $t=0$ and then use this value for $t \neq 0$ :

$$
a_{s}=a_{e}^{*}(t=0) \frac{\Gamma a_{0}}{d+v_{s}} \exp \left[\left(\frac{-s-i \Delta \omega}{2}\right) t\right] \sinh (d t) .
$$

[Notice that if the coupling were zero $(\Gamma \rightarrow 0) a_{s}$ would remain zero.]

To obtain $a_{e}^{*}$, the simplest course is to solve Eq. (B1), thus

$$
\begin{aligned}
a_{e}^{*}(t)= & \frac{\exp (i \Delta \omega t)}{\Gamma a_{0}} a_{e}^{*}(t=0) \frac{\Gamma a_{0}}{d+v_{s}} \\
& \times\left[\left(\frac{d}{d t}\right)+v_{s}\right]\left[\frac{1}{2} \exp \left(-\frac{i \Delta \omega+s}{2}+d\right) t\right. \\
& \left.-\frac{1}{2} \exp \left(-\frac{i \Delta \omega+s}{2}-d\right) t\right]
\end{aligned}
$$

Carrying out these operations and the obvious simplifications produces a result whose behavior both, asymptotic and initial, is clear:

$$
\begin{aligned}
a_{0}^{*}(t)= & a_{0}^{*}(t=0) \exp \left(\frac{i \Delta \omega-s}{2} t\right) \\
& \times\left(\cosh (d t)-\frac{i \Delta \omega+s}{2\left(d+v_{s}\right)} \sinh (d t)\right) .
\end{aligned}
$$

Note that, if one checks (as one should) that Eq. (B2) is also satisfied by these solutions, it will be necessary to invoke a result from the dispersion equation solution $\mathrm{Eq}$. (B6), namely that $d^{2}-\left(v_{e}-v_{s}+i \Delta \omega\right)^{2} / 4$ is equal to $\Gamma^{2}\left|a_{0}\right|^{2}$.

This more or less cosh-like and sinh-like behavior for the mode pair is clear in the logarithmic presentations of Fig. 4. For more restricted ranges, such as for the first simulation, one can test the fit of these linear mode pair solutions to the EVF or three-oscillator results without requiring decades of exponential behavior.

${ }^{1}$ D. W. Forslund, J. M. Kindel, and E. L. Lindman, Phys. Fluids 18, 1017 (1975).

${ }^{2}$ T. Tajima and J. M. Dawson, Phys. Rev. Lett. 43, 267 (1979).

${ }^{3}$ C. Joshi, T. Tajima, J. M. Dawson, H. A. Baldis, and N. A. Ebrahim, Phys. Rev. Lett. 47, 1285 (1981).

${ }^{4}$ C. Darrow, W. B. Mori, T. Katsouleas, C. Joshi, D. Umstadter, and C. E. Clayton, IEEE Trans. Plasma Sci. PS-15, 707 (1987).

${ }^{5}$ K. Estabrook, W. L. Kruer, and F. A. Williams, Phys. Fluids 31, 372 (1988).

${ }^{6}$ A. Ghizzo, P. Bertrand, T. W. Johnston, and M. Shoucri, to appear in J. Comput. Phys.

${ }^{7}$ A. Ghizzo, P. Bertrand, M. Shoucri, T. W. Johnston, and M. R. Feix, J. Comput. Phys. 90, 431 (1990).

${ }^{8}$ P. Bertrand, A. Ghizzo, T. W. Johnston, M. Shoucri, E. Fijalkow, and M. Feix, Phys. Fluids B 2, 1028 (1990).

${ }^{9}$ D. W. Forslund, J. M. Kindel, and E. L. Lindman, Phys. Fluids 18, 1002 (1975).

${ }^{10}$ W. B. Mori, IEEE Trans. Plasma Sci. PS-15, 88 (1987).

${ }^{11}$ M. N. Rosenbluth and C. S. Liu, Phys. Rev. Lett. 29, 701 (1972).

${ }^{12}$ W. Mori, Phys. Rev. A 44, 5118 (1991).

${ }^{13}$ S. Aithal, P. Lavigne, H. Pépin, T. W. Johnston, and K. Estabrook, Phys. Fluids 30, 3832 (1987). 